\title{
Review: famotidine, pizotifen, cognitive behavioural therapy, and peppermint may be effective in recurrent abdominal pain
}

Weydert JA, Ball TM, Davis MF. Systematic review of treatments for recurrent abdominal pain. Pediatrics 2003;111:e1-11.

QUESTION: In children, what is the effectiveness of treatments for recurrent abdominal pain (RAP)?

\section{Data sources}

Studies were identified by searching Medline (1966 to 2001), the Cochrane Clinical Trial registry, the Cochrane Database of Systematic Reviews, AMED, PsycINFO, and PubMed via the National Center for Complementary and Alternative Medicine; and by reviewing bibliographies of relevant articles using terms including recurrent abdominal pain, functional abdominal pain, irritable bowel syndrome, children, and alternative therapies.

\section{Study selection}

English language randomised controlled trials (RCTs) were selected if they were done on children aged $\leq 18$ years with a diagnosis of RAP. Studies not on functional gastrointestinal disorders, and those lacking a control group were excluded.

\section{Data extraction}

Data were extracted on participants' characteristics and heterogeneity, study quality and design, interventions, outcomes, and a study's adherence to the standardised case definition of RAP ( $\geq 3$ episodes of abdominal pain occurring over $\geq 3 \mathrm{mo}$ and severe enough to interfere with normal activities).

\section{Main results}

10 trials met the inclusion criteria and all studies stated that they were blinded. 2 studies were of pharmacological interventions. Of these, 1 study (25 children) suggested that famotidine might be superior to placebo in functional abdominal pain with dyspepsia. 1 study (14 children) suggested that pizotifen, used prophylactically, might be more effective than placebo in abdominal migraines. 4 studies were of dietary interventions in children with RAP. 1 study (52 children) suggested that fibre reduced frequency of pain attacks more than did placebo and another study of fibre in 40 children gave inconclusive data. 1 study (39 children) suggested that a lactose free diet is unlikely to improve RAP symptoms. Another study (69 children) of lactose avoidance had inconclusive data due to a high $(45 \%)$ dropout rate. 2 methodologically strong studies (in 16 and 44 children, respectively) showed that cognitive behavioural therapy reduced or eliminated pain without side effects or symptom substitution in children with RAP. 1 study (61 children) suggested that cognitive behavioural therapy and biofeedback plus fibre may be more effective than fibre alone. 1 study (50 children) suggested that a $\mathrm{pH}$ dependent peppermint oil capsule reduced pain during the acute phases of RAP and irritable bowel syndrome.

\section{Conclusions}

In children with recurrent abdominal pain, famotidine, pizotifen, cognitive behavioural therapy, biofeedback, and peppermint oil may be effective. Studies of dietary fibre give inconclusive data and lactose free diets are unlikely to be effective.

\section{COMMENTARY}

Because RAP is a frequent but mostly non-organic symptom in school children, ${ }^{1}$ it is important to review treatments for RAP systematically. The methodology for identifying RCTs was scientifically sound, although it was clear that treatments and results would be varied given the heterogeneous nature of this group of functional disorders. The findings from this systematic review may not have disclosed the whole truth, and more high quality studies with larger populations are needed using the Rome II criteria to better subtype functional abdominal disorders and to target treatments.

The main practice recommendations are not weighted enough in the conclusions. Children with RAP had the largest benefit from mind body therapies, and, in subgroups of children with specific gastrointestinal symptoms, from the pharmaceuticals famotidine, pizotifen, or peppermint oil. These findings are not surprising considering the multiple interactions between the central and the enteral nervous system (ie, the brain gut axis). Comparing these systems with a computer network, these interactions are easily explainable to children who use computers almost daily and have experienced non-functioning programmes now and then. It is also important to consider the role of genetics, because many parents also have stress sensitive guts. ${ }^{2}$ Dietary changes may be more important than the RCTs have shown. Lactose intolerance may be more relevant in distinct populations (eg, in groups with Mediterranean, African, or Asian origin), and fructose malabsorption (eg, from apple juice) is not mentioned in RAP. The detection of Helicobacter pylori has led to some enthusiasm for this as a cause of RAP. However, only a minority of about $10 \%$ of children with RAP benefit from eradication of Helicobacter pylori. ${ }^{4}$ Coeliac disease is much more prevalent worldwide than thought previously. Considering the oligosymptomatic "hidden" cases (eg, the iceberg phenomenon), more children with RAP may be detected who suffer from gluten intolerance. Professor Dr Klaus-Michael Keller, MD Deutsche Klinik fur Diagnostik Aukammallee, Wiesbaden, Germany

1 Apley J, Naish N. Recurrent abdominal pains: a field survey of 1,000 school children. Arch Dis Child 1958;33:165-170.

2 Levy RL, Jones KR, Whitehead WE, et al. Irritable bowel syndrome in twins: heredity and social learning both contribute to etiology. Gastroenterology 2001;121:799-804.

3 Ashorn M, Mäki M, Ruuska T, et al. Upper gastrointestinal endoscopy in recurrent abdominal pain of childhood. J Pediatr Gastroenterol Nutr 1993;16:273-7.

4 Gold BD, Colletti RB, Abbott M, et al. Helicobacter pylori infection in children: recommendations for diagnosis and treatment. J Pediatr Gastroenterol Nutr 2000;31:490-7. 